\title{
AN ASSESSMENT OF COLLABORATIVE GOVERNANCE IN NETWORKING FOR CONTENTS TOURISM
}

\author{
Satoshi Ishida \\ Department of Public Policy, Faculty of Regional Design and Development, \\ University of Nagasaki, Japan \\ (st.ishida@sun.ac.jp)
}

\begin{abstract}
The tourism industry has been recognised as a potential development mechanism in local communities facing various changes and challenges. In recent years, content tourism, which is the utilisation of lands associated with works appearing in subcultures and other content works for which Japan has attracted international attention, as a tourism resource. However, previous studies have not revealed much about the practice of contents tourism, especially those that examine it from the perspective of collaborative governance. This article presents a case study of a cross-sectoral organisational network designated to promote contents tourism in Sasebo, Nagasaki. This paper briefly introduces the topics of collaborative governance and contents tourism, and then the network for cross-sectoral collaboration and the research method. As a result, findings are discussed in terms of key elements of collaborative governance, aspects of the network formation and development process and features of the Sasebo's context that may influence the network development. In conclusion, this paper focuses on the role of key stakeholders in formatting a network in the context of content tourism. The struggle to formulate a collaborative governance approach for effective content tourism may bring positive socio-economic benefits to the city and similar declining cities in Japan. As this approach is conceptually tentative and in its developmental stage in theory and practice, it needs to be complemented by additional research findings from empirical case studies conducted with broader and more diverse stakeholder involvement.
\end{abstract}

KEYWORDS: Contents Tourism, Collaborative Governance, Networking, Assessment, Sasebo

\section{PURPOSE AND BACKGROUND}

The tourism sector in Japan have been exploring ways to promote and assess progress towards contents tourism. These efforts have reflected growing interest and recognition of contents tourism, to be more successful they need to integrate both government sectors and private sectors through cross-sectoral collaboration and its network that can enhance the level of stakeholder participation in tourism development. The cross-sectoral collaboration with broad stakeholder has emerged recently as an important topic in the public policy literature.

Today, in Japan, there are considerable amount and array of contents tourism-related activities taking place in the context of cross-sectoral networks of diverse individuals and organisations. Given the importance of diverse entities working together to accomplish common objectives and produce shared value, such systems have been identified as comprising a new form of "collaborative governance".

Available literature on collaborative governance in the tourism sector provides much useful information on how collaborative governance systems can be designed and managed so as to work effectively. However, there is still much to be learned from studying a diverse array of these efforts to better understand how they function and what makes it work successfully. 
This paper focuses on a case of collaborative governance recently initiated to promote contents tourism activities in Sasebo, Nagasaki Prefecture, Japan. Between 2018 and 2019, the Sasebo Project Team for Regional Revitaliszation (SPTR2), as a cross-sectoral network of stakeholders.

\section{METHODOLOGY}

As a research methodology, this study is a qualitative case study based on (structured and semi-structured) interviews with SPTR2 stakeholders and document analysis. The paper contextualises the early stages of content tourism development in Sasebo and identifies key management issues that need to be addressed to capture future opportunities. Furthermore, it reflects on the recent characteristics of content tourism in Japan as a potential option to promote regional revitalisation.

\section{FINDINGS}

According to interviews with SPTR2 members, one problem has been the spread of content tourism related activities, which has increased the awareness of the content's tourism to residents and tourists. However, there have been reports of cases where people have used characters in games without the permission of the content holders and have been severely warned by the content rights holders. There are also reports of cases of severe warnings from SPTR2.

Therefore, in the future, it is necessary to establish a compliance system within the region, as well as rules or guidelines regarding the use of content tourism-related resources (e.g., copyright pictures of characters). In Sasebo, many of key players in the tourism sector have long-standing formal and informal, as well as personal and professional relationships, which provide a foundation of trust and a source of social capital for the "start-up" of SPTR2.

In order for SPTR2 to develop into a significant system with a meaningful impact on sustainable contents tourism in Sasebo, it is necessary to build quality relationships, create an environment of trust and openness, and establish the conditions for "authentic dialogue" as the basis for collective decision-making and joint implementation. Ongoing effort will be needed.

\section{CONCLUSION}

To conclude the analysis, from a comparative perspective, this paper examines what features of the Sasebo context are particularly important for considering SPTR2 and the conditions necessary for its success.

First, there is a kind of paradox regarding the formation of networks in Sasebo. There are already a number of associations, federations, and networks in the tourism sector. Some stakeholders seem to be willing to actively participate in tourism-related events and become part of some kind of affiliation group.

However, one of the main challenges in promoting the regional integration of tourism is the need for a "paradigm shift from 'me' to 'us' (Lima 2009). The challenge of overcoming the tendency towards self-interest may be nearly universal, but according to the authors' observations, there is still a lack of discussion in terms of what is needed for the network as a whole to benefit. Given the potential for synergies in the tourism sector, this paradigm shift could provide the basis for identifying win-win solutions that simultaneously benefit multiple diverse stakeholders. 


\section{CONTRIBUTION/PRACTICAL IMPLICATIONS}

The significance of the research is that it will help to build cross-sectoral governance in effective content tourism (industry), especially in cities where access to content industry and content business is limited.

\section{ACKNOWLEDGEMENTS}

The authors would like to thank the members of SPTR2 for their interview assistance in writing this paper.

\section{REFERENCES}

Ansell, C., \& Gash, A. (2008). Collaborative governance in theory and practice. Journal of Public Administration Research and Theory, 18(4), 543-571.

Bryson, J. M., Crosby, B. C., \& Stone, M. M. (2006). The design and implementation of cross-sector collaborations: Propositions from the literature. Public Administration Review, 66(1), 44-55.

Seaton, P., Yamamura, T., Sugawa-Shimada, A., \& Jang, K. (2016). Contents tourism in Japan: Pilgrimages to "Sacred Sites" of popular culture. Cambria Press. 